EPJ Web of Conferences 71, 00094 (2014)

DOI: 10.1051/epjconf/20147100094

(C) Owned by the authors, published by EDP Sciences, 2014

\title{
Dark Matter Signals in the gamma-ray sky
}

\author{
Aldo Morselli ${ }^{1, a}$ \\ ${ }^{1}$ INFN Roma Tor Vergata
}

\begin{abstract}
Detection of gamma rays and cosmic rays from the annihilation or decay of dark matter particles is a promising method for identifying dark matter, understanding its intrinsic properties, and mapping its distribution in the universe. I will review recent results from the Fermi Gamma-ray Space Telescope and other space-based experiments, and highlight the constraints these currently place on particle dark matter models. I will also discuss the prospects for indirect searches to robustly identify or exclude a dark matter signal using upcoming data.
\end{abstract}

One of the major open issues in our understanding of the Universe is the existence of an extremelyweakly interacting form of matter, the Dark Matter (DM), supported by a wide range of observations including large scale structures, the cosmic microwave background and the isotopic abundances resulting from the primordial nucleosynthesis. The DM might be detected indirectly through anomalous cosmic rays (CRs) produced by their annihilations in the galactic center, galactic halo or extragalactic structures such as dwarf spheroidal galaxies and clusters. These annihilations will produce $\gamma$-rays or antimatter, and fluxes of these particles can be measured in space-based detectors such as Fermi-LAT ( $\gamma$-rays) and PAMELA or AMS (antimatter). $\gamma$-rays can also be measured in Cherenkov telescopes such as MAGIC, HESS or VERITAS. On the other hand, neutrino telescopes might detect WIMPs passing through the Sun and/or Earth. They may be slowed below escape velocity by elastic scattering. Then, the annihilation of WIMPs accumulated due to gravitational effects produces energetic neutrinos that can be detected in underground (Super-Kamiokande), underwater (ANTARES) and under-ice (IceCube) experiments (see figure 1). The direct measurement of Cosmic Ray started for INFN around the 1989 with the beginning of the activity of the WiZard Collaboration, described in [1] and [2]. Beginning from 1995, the WiZard Collaboration and the AMS Collaboration begun to launch also space detectors.

\subsection{The Cosmic Ray Electron spectrum}

The experimental information available on the Cosmic Ray Electron (CRE) spectrum has been dramatically expanded with a high precision measurement of the electron spectrum from $7 \mathrm{GeV}$ to $1 \mathrm{TeV}$ by the Fermi LAT [3-5]. The spectrum shows no prominent spectral features and it is significantly harder than that inferred from several previous experiments [6, 7].

Recently the Fermi-LAT collaboration performed a direct measurement of the absolute $e^{+}$and $e^{-}$ spectra, and of their fraction [8]. As the Fermi-LAT does not carry a magnet, analysis took advantage of the fact that due to its magnetic field, the Earth casts a shadow in electron or positron fluxes in

\footnotetext{
a e-mail: aldo.morselli@roma2.infn.it
} 


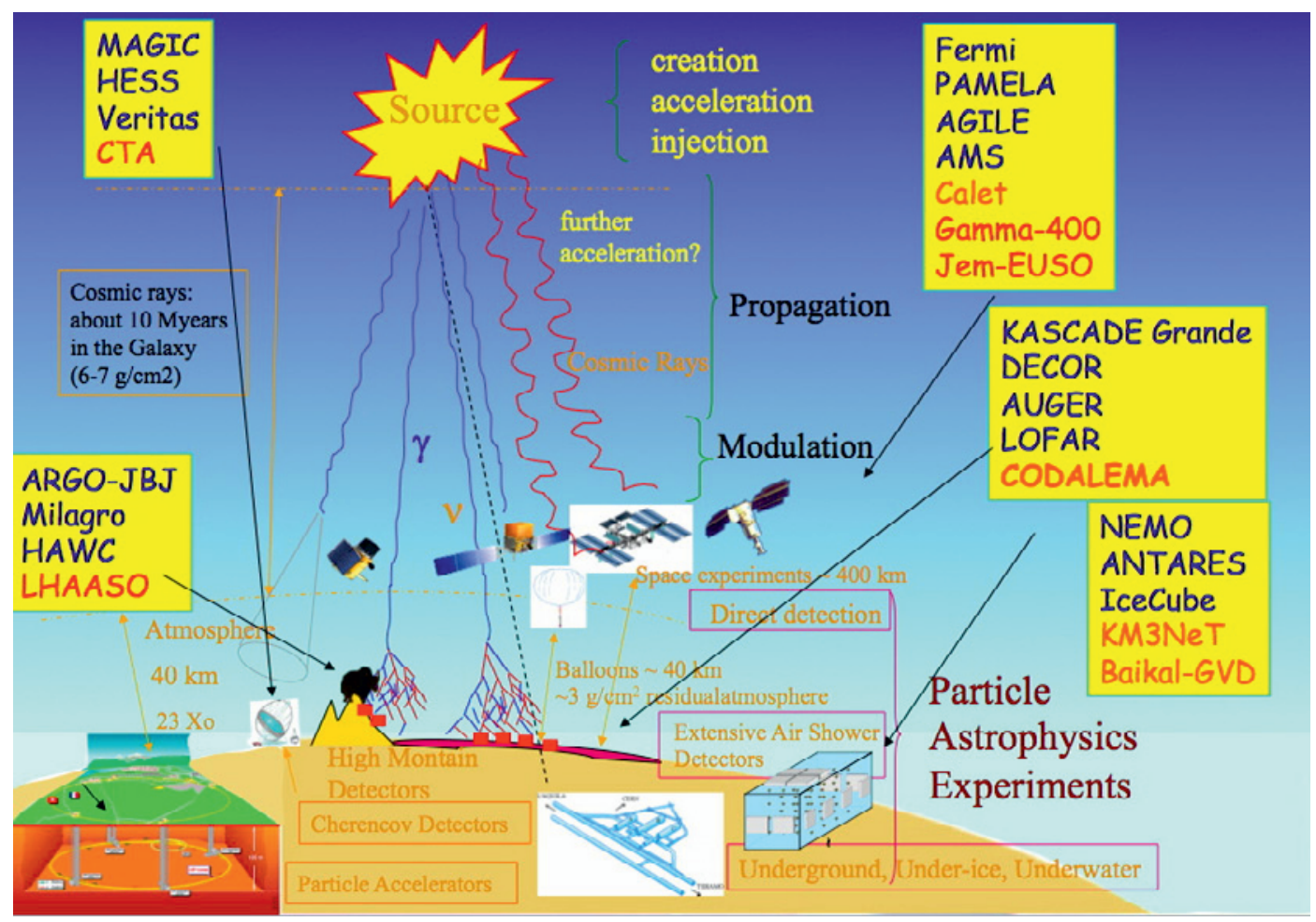

Figure 1. Indirect, direct and accellerator experiments for the search of the nature of dark matter (the future one's are in red)

precisely determined regions. As a result, this measurement confirmed a rise of the positron fraction observed by PAMELA, between 20 and $100 \mathrm{GeV}[9,10]$ and determine for the first time that it continues to rise between 100 and $200 \mathrm{GeV}$ (see Fig.2). These measurements show that a new component of $e^{+}$and $e^{-}$are needed with a peak at about $1 \mathrm{TeV}$. The temptation to claim the discovery of dark matter from detection of electrons and positrons from annihilation of dark matter particles is strong but there are competing astrophysical sources, such as pulsars, that can give a strong flux of primary positrons and electrons (see [11] and references therein). At energies between $100 \mathrm{GeV}$ and $1 \mathrm{TeV}$ the electron flux reaching the Earth may be the sum of an almost homogeneous and isotropic component produced by Galactic supernova remnants and the local contribution of a few pulsars with the latter expected to contribute more and more significantly as the energy increases.

If a single nearby pulsar give the dominant contribution to the extra component a large anisotropy and a small bumpiness should be expected; if several pulsars contribute the opposite scenario is expected. So far no positive detection of Cosmic Ray Electron (CRE) anisotropy was reported by the Fermi-LAT collaboration, but some stringent upper limits were published [12] and the pulsar scenario is still compatible with these upper limits.

The AMS-02 collaboration presented the measurement of the positron fraction [13] that confim the positron ratio rise observed by PAMELA and Fermi Large Area Telescope (Fermi-LAT ) and extend it up to $350 \mathrm{GeV}$ (see figure 2).

Forthcoming measurements from AMS-02 and CALET are expected to reduce drastically the uncertainties on the propagation parameters by providing more accurate measurements of the spectra 


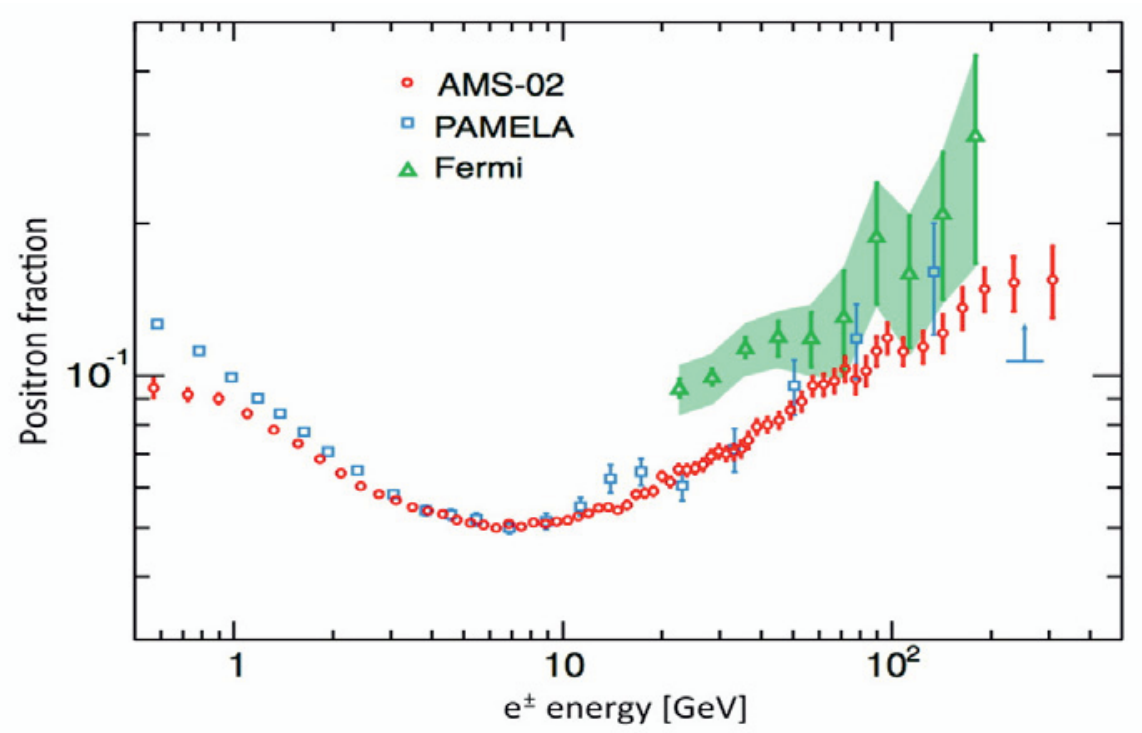

Figure 2. Positron fraction measured by the Fermi-LAT [8], PAMELA [9, 10] and AMS-02 [13]. The FermiLAT statistical uncertainty is shown with error bars and the total (statistical plus systematic uncertainty) is shown as a shaded band

of the nuclear components of CR. Fermi-LAT and those experiments are also expected to provide more accurate measurements of the CRE spectrum and anisotropy looking for features which may give a clue of the nature of the extra component.

\subsection{The Galactic center}

The Galactic center (GC) is expected to be the strongest source of $\gamma$-rays from DM annihilation, due to its coincidence with the cusped part of the DM halo density profile [14], [15]. A preliminary analysis of the data, taken during the first 11 months of the Fermi satellite operations is presented in [17], [18]. The diffuse gamma-ray backgrounds and discrete sources, as we know them today, can account for the large majority of the detected gamma-ray emission from the Galactic Center. Nevertheless a residual emission is left, not accounted for by the above models [17], [18].

Improved modeling of the Galactic diffuse model as well as the potential contribution from other astrophysical sources (for instance unresolved point sources) could provide a better description of the data. Analyses are underway to investigate these possibilities.

Although several astrophysical processes at work in the crowded GC region make it extremely difficult to disentangle the DM signal from conventional emissions, the DM-induced gamma-ray emission is expected to be so large there that the search is still worthwhile. Furthermore, the DM density in the GC may be larger than what is typically obtained in $N$-body cosmological simulations.

The analysis of the constraints that can be obtained for generic DM candidates from Fermi-LAT inner Galaxy gamma-ray measurements assuming some specific DM distributions is shown in figure 3 for the $b \bar{b}$ channel as an example [19]. The approach is conservative, requiring simply that the expected DM signal does not exceed the gamma-ray emission observed by the Fermi-LAT in an optimized region around the GC. The region is chosen in such a way that the $\mathrm{S} / \mathrm{N}$ ratio is maximized. 


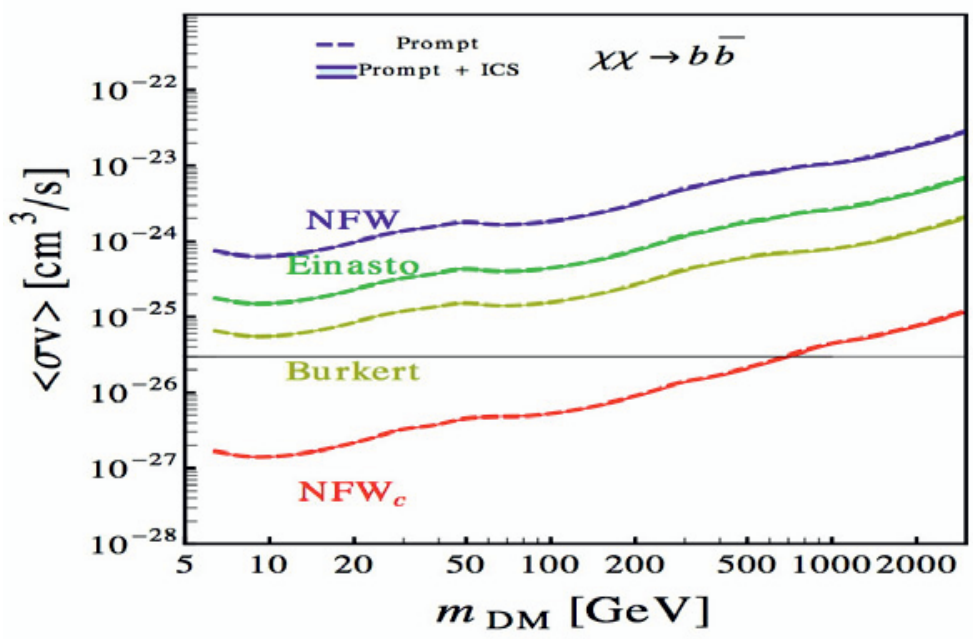

Figure 3. $3 \sigma$ upper limits on the annihilation cross-section of models in which DM annihilates into $b \bar{b}$ for four DM density profiles [19]. The horizontal line corresponds to the expected value of the thermal cross-section for a generic WIMP candidate.

The constraints obtained in the likely case that the collapse of baryons to the Galactic Center is accompanied by the contraction of the dark matter are strong. In particular, for $b \bar{b}$ and $\tau^{+} \tau^{-}$or $W^{+} W^{-}$ dark matter annihilation channels, the upper limits on the annihilation cross section imply that the thermal cross section is excluded for a Weakly Interacting Massive Particle (WIMP) mass smaller than about 700 and $500 \mathrm{GeV}$, respectively. For the $\mu^{+} \mu^{-}$channel, where the effect of the inverse Compton scattering is important, depending on models of the Galactic magnetic field the exclusion of the thermal cross-section is for a WIMP mass smaller than about 150 to $400 \mathrm{GeV}$. The upper limits on the annihilation cross section of dark matter particles obtained are two orders of magnitude stronger than without contraction [19].

\subsection{Dwarf galaxies}

Dwarf satellites of the Milky Way are among the cleanest targets for indirect dark matter searches in gamma-rays. They are systems with a very large mass/luminosity ratio (i.e. systems which are largely DM dominated). The LAT detected no significant emission from any of such systems and the upper limits on the $\gamma$-ray flux allowed us to put very stringent constraints on the parameter space of well motivated WIMP models [20, 21].

A combined likelihood analysis of the 15 most promising dwarf galaxies, based on 4 years of data and pushing the limits below the thermal WIMP cross section for low DM masses (below a few tens of $\mathrm{GeV}$ ), has been recently performed [22].

The derived $95 \%$ C.L. upper limits on WIMP annihilation cross sections for different channels are shown in figure 4 . The most generic cross section $\left(\sim 3 \cdot 10^{-26} \mathrm{~cm}^{3} \mathrm{~s}^{-1}\right.$ for a purely s-wave cross section) is plotted as a reference. These results are obtained for NFW profiles but for cored dark matter profile the J-factors for most of the dSphs would either increase or not change much so these results includes J-factor uncertainties [21,22]. With the present data we are able to rule out large parts of the parameter space where the thermal relic density is below the observed cosmological dark matter 


\section{ICNFP 2013}

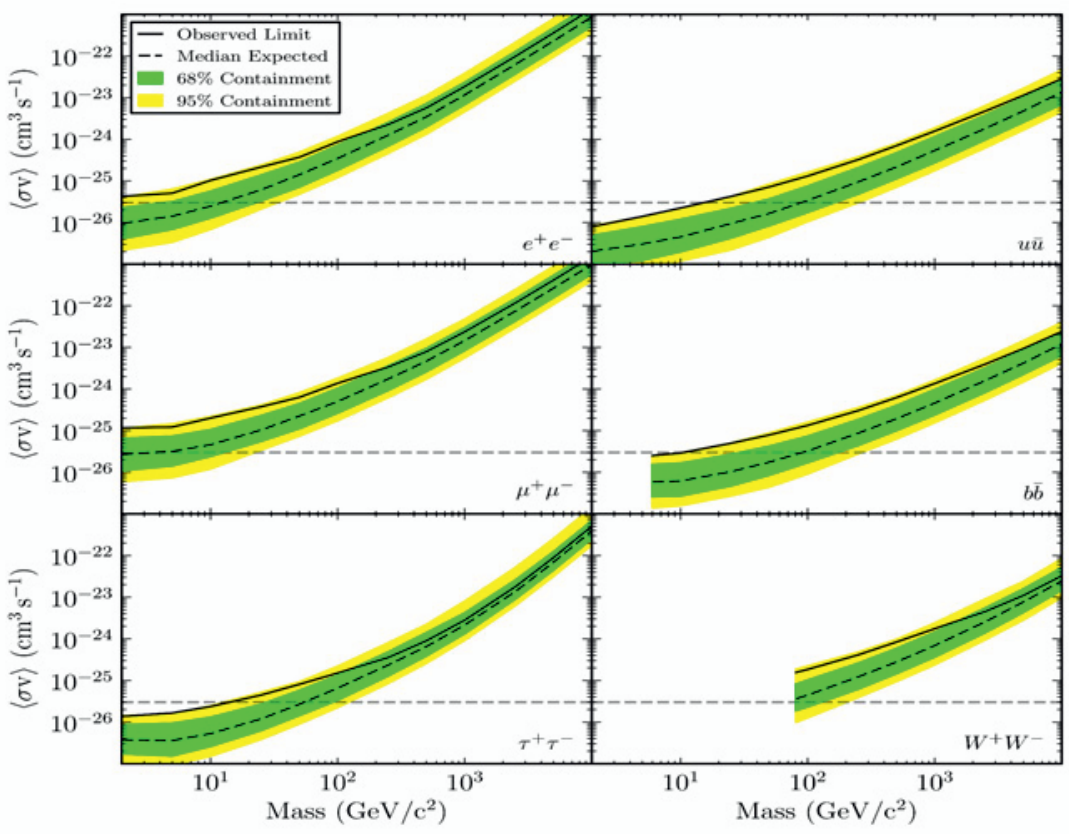

Figure 4. Constraints on the dark matter annihilation cross section at 95\% C.L. derived from a combined analysis of 15 dwarf spheroidal galaxies assuming an NFW dark matter distribution (solid line). In each panel bands represent the expected sensitivity as calculated by repeating the combined analysis on 300 randomly-selected sets of blank fields at high Galactic latitudes in the LAT data. The positions and widths of the expected sensitivity bands reflect the range of statistical fluctuations expected both from the LAT data and from the stellar kinematics of the dwarf galaxies. The most significant excess in the observed limits occurs for the $b \bar{b}$ channel between 10 $\mathrm{GeV}$ and $25 \mathrm{GeV}$ with $\mathrm{TS}=8.7$.

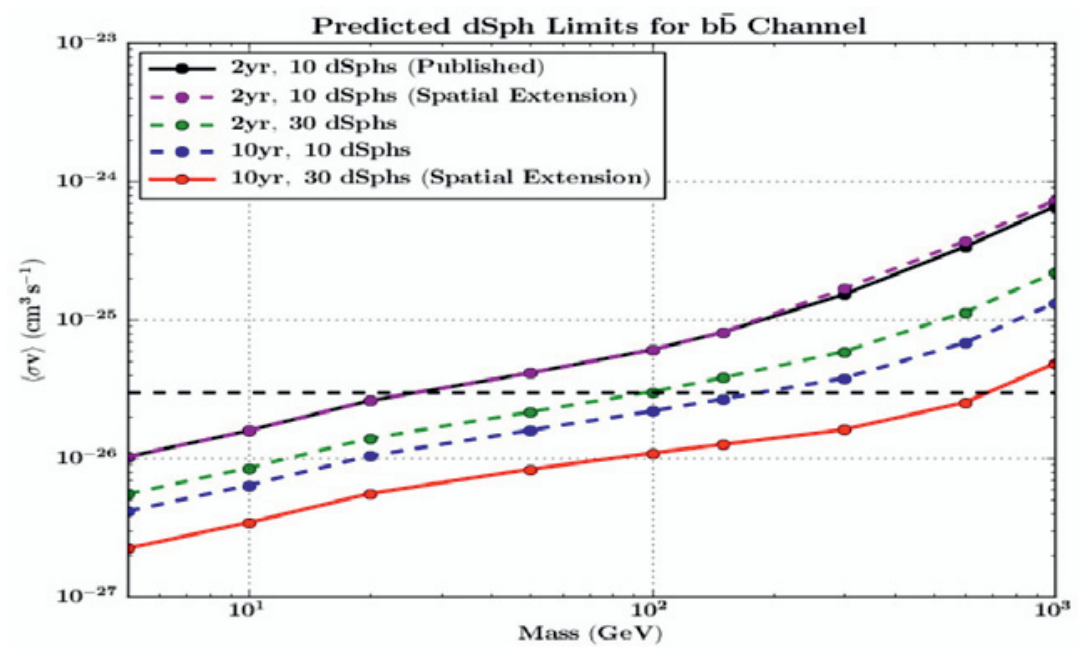

Figure 5. Predicted upper limits in 10 years for the $b \bar{b}$ channel 


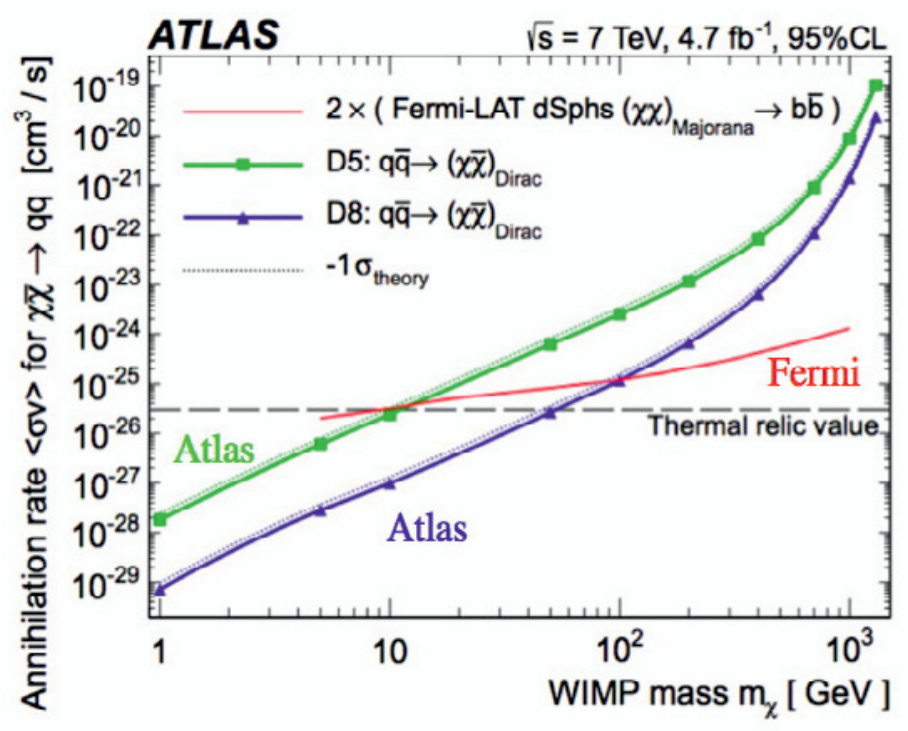

Figure 6. Inferred ATLAS 95\% CL limits on WIMP annihilation rates versus mass The thick solid lines are the observed limits excluding theoretical uncertainties. The ATLAS limits are for the four light quark flavours assuming equal coupling strengths for all quark flavours to the WIMPs. For comparison, high-energy gamma-ray limits from observations of Galactic satellite galaxies with the Fermi-LAT experiment for Majorana WIMPs are shown. The Fermi-LAT limits are scaled up by a factor of two to make them comparable to the ATLAS Dirac WIMP limits. All limits shown here assume $100 \%$ branching fractions of WIMPs annihilating to quarks. The horizontal dashed line indicates the value required for WIMPs to make up the relic abundance set by the WMAP measurement.

density and WIMPs are dominantly produced non-thermally, e.g. in models where supersymmetry breaking occurs via anomaly mediation. These $\gamma$-ray limits also constrain some WIMP models proposed to explain the Fermi-LAT and PAMELA $e^{+} e^{-}$data, including low-mass wino-like neutralinos and models with $\mathrm{TeV}$ masses pair-annihilating into muon-antimuon pairs.

Future improvements (apart from increased amount of data) will include an improved event selection with a larger effective area and photon energy range, and the inclusion of more satellite galaxies. In figure 5 are shown the predicted upper limits in the hypothesis of 10 years of data instead of 2; 30 dSphs instead of ten (supposing that the new optical surveys will find new dSph); spatial extension analysis (source extension increases the signal region at high energy $E \geq 10 \mathrm{GeV}, M \geq 200 \mathrm{GeV}$ ).

Note that thiese searches are really complementary and at the same quantitative level of the searches that can be performed at LHC (see for example figure 6 from [23]).

\subsection{Gamma-ray lines}

A line at the WIMP mass, due to the $2 \gamma$ production channel, could be observed as a feature in the astrophysical source spectrum [16]. Such an observation would be a "smoking gun" for WIMP DM as it is difficult to explain by a process other than WIMP annihilation or decay and the presence of a feature due to annihilation into $\gamma Z$ in addition would be even more convincing. 


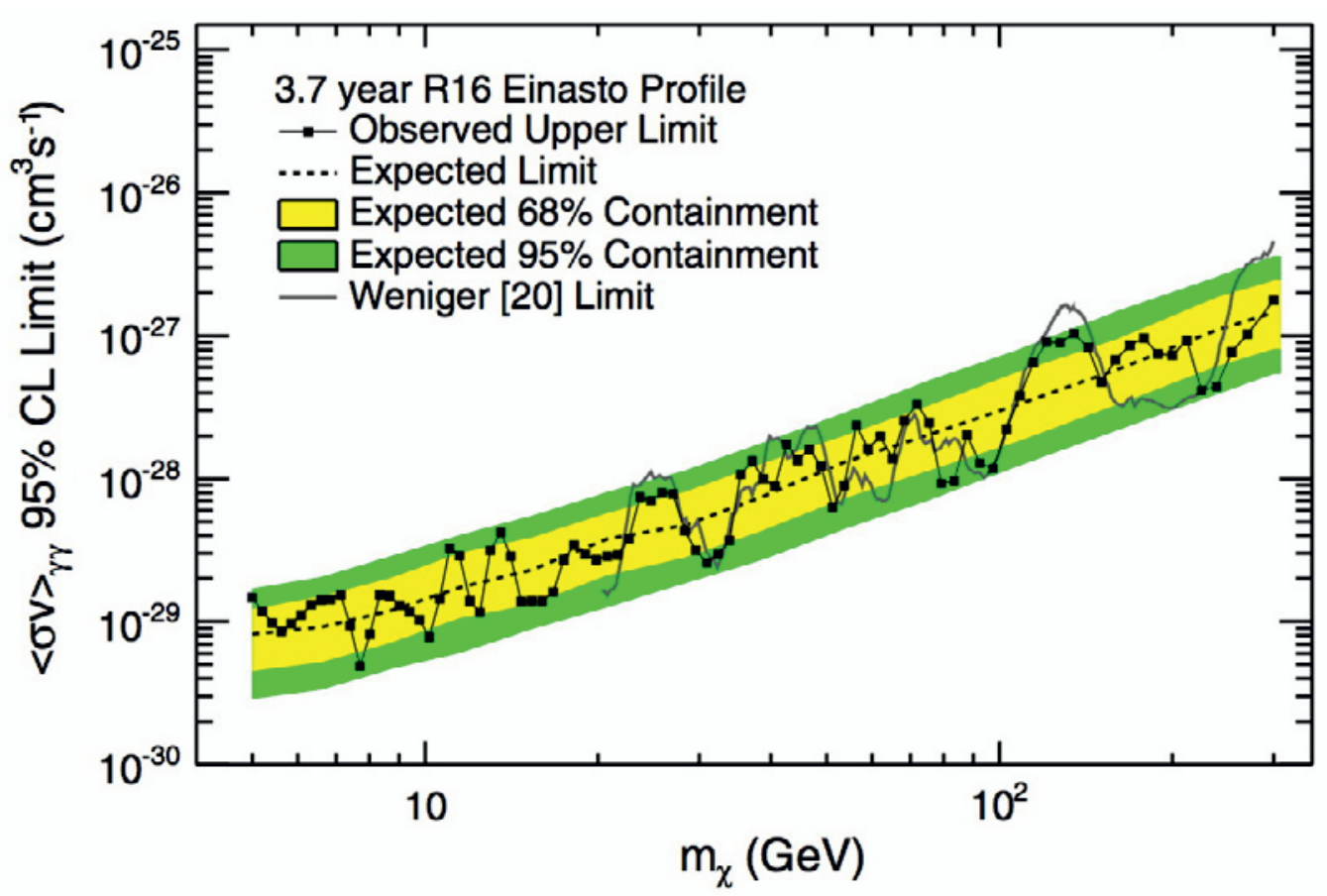

Figure 7. Dark matter annihilation $95 \% \mathrm{CL}$ cross section upper limits into $\gamma \gamma$ for the Einasto profile for a circular region of interest (ROI) with a radius $\mathrm{R}_{G C}=16^{\circ}$ centered on the GC with $|b|<5^{\circ}$ and $|l|>6^{\circ}$ masked.

No significant evidence of gamma-ray line(s) has been found in the first two years of data from 7 to $200 \mathrm{GeV}$ [24] (see also [25]).

Recently, the claim of an indication of line emission in Fermi-LAT data [26, 27] has drawn considerable attention. Using an analysis technique similar to [25], but doubling the amount of data as well as optimizing the region of interest for signal over square-root of background, [26] found a (trial corrected) $3.2 \sigma$ significant excess at a mass of $\sim 130 \mathrm{GeV}$ that, if interpreted as a signal would amount to a cross-section of about $\left\langle\sigma v>\sim 10^{-27} \mathrm{~cm}^{3} \mathrm{~s}^{-1}\right.$.

The signal is found to be concentrated on the Galactic Centre with a spatial distribution consistent with an Einasto profile [28]. This is marginally compatible with the upper limit presented in [24]. In the analysis of the 4 year data the Fermi LAT team has improved over the two year paper in three important aspects: i) the search was performed in five regions of interest optimized for DM search under five different assumptions on the morphology of the DM signal, ii) new improved data set (pass 7 reprocessed) was used, as it corrects for loss in calorimeter light yield due to radiation damage during the four years of the Fermi mission and iii) point spread function (PDF) was improved by adding a 2nd dimension to the previously used triple Gaussian PDF model, leading to a so called '2D' PDF (such procedure is shown to increase the sensitivity to a line detection by $15 \%$ ). In that analysis [29] no globally significant lines have been fond and new limits to this DM annihilation channel were set (see figure 7). In a close inspection of the $130 \mathrm{GeV}$ feature it was found that indeed there exist a $135 \mathrm{GeV}$ signal at $4.01 \sigma$ local significance, when a '1D' PSF and old data sets were used (consistently with what $[26,27]$ have found). However, the significance drops to $3.35 \sigma$ (local, or $\leq 2 \sigma$ global significance once trials factors are taken into account). In addition, a weaker signal is 


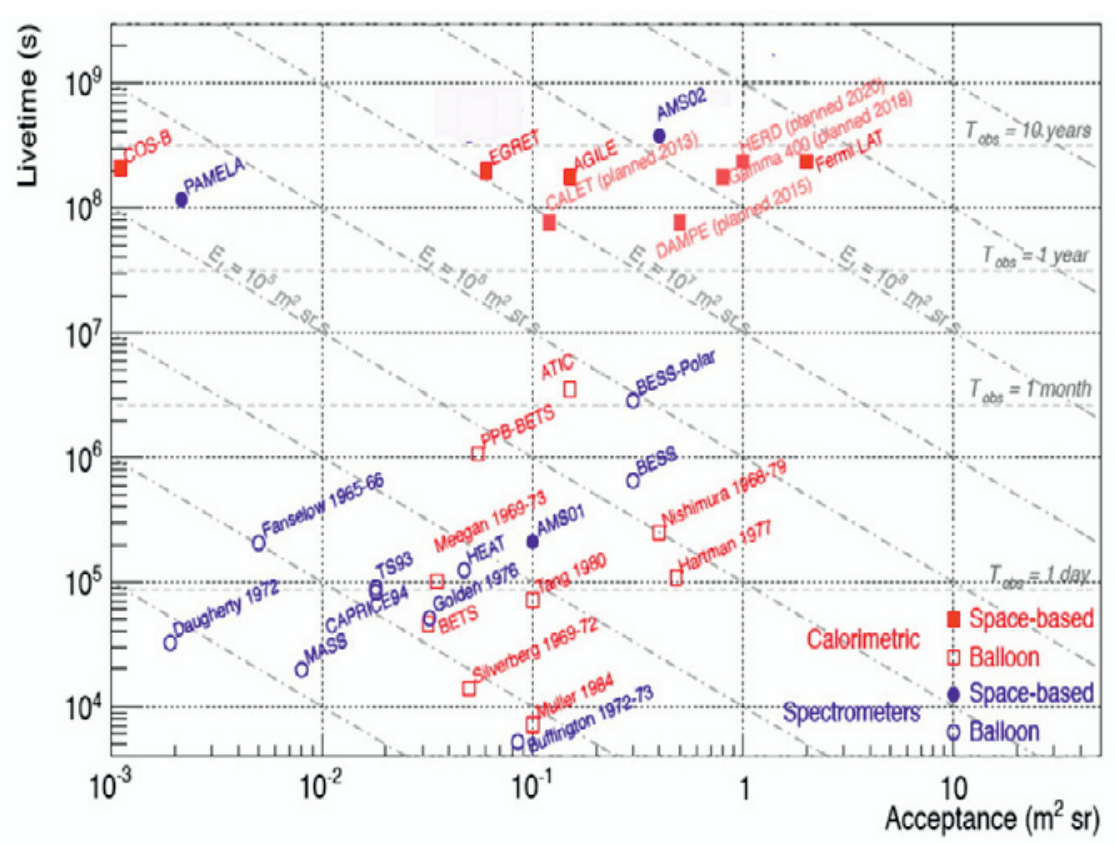

Figure 8. Livetime versus acceptance for present and future detectors in gamma-ray astrophysics

found at the same energy in the control sample (in the Earth limb), which might point to a systematics effect present in this data set. In order to examine this possibility weekly observations of the Limb are scheduled, and a better understanding of a nature of the excess in the control sample should be available soon. A new version of the event-level reconstruction and analysis framework (called Pass 8 ) is foreseen soon from the Fermi LAT collaboration. With this new analysis software they should increase the efficiency of the instrument at high energy and have a data set based on independent event analysis thus gaining a better control of the systematic effects.

\section{Conclusions}

Fermi-LAT turned five years in orbit on June, 2013, and it is definitely living up to its expectations in terms of scientific results delivered to the community. The mission is planned to continue at least three more years (likely until 2018) with many remaining opportunities for discoveries.

In figure 8 it is shown the livetime versus acceptance for present and future detectors in gammaray astrophysics. It can be see tht Fermi-LAT has the largest acceptance even if compared with future project.

In figure 9 it is shown the time of operation and energy range of space X-ray satellite and gammaray experiments . Note that AGILE and Fermi-LAT have covered an interval not covered by any other experiments. Note also the number of other experiments in other frequencies that are allowing extensive multifrequency studies. The angular resolution and energy resolution achievable in gamma ray astrophysics is still lower to what is desirable and achievable in other band. With the forthcoming Gamma-400 experiment we hope to try to fill this gap [30] expecially if the front part of the decector will be tuned for the low energy band (below $100 \mathrm{MeV}$ ) as described in the Gamma-Light project [31]. 


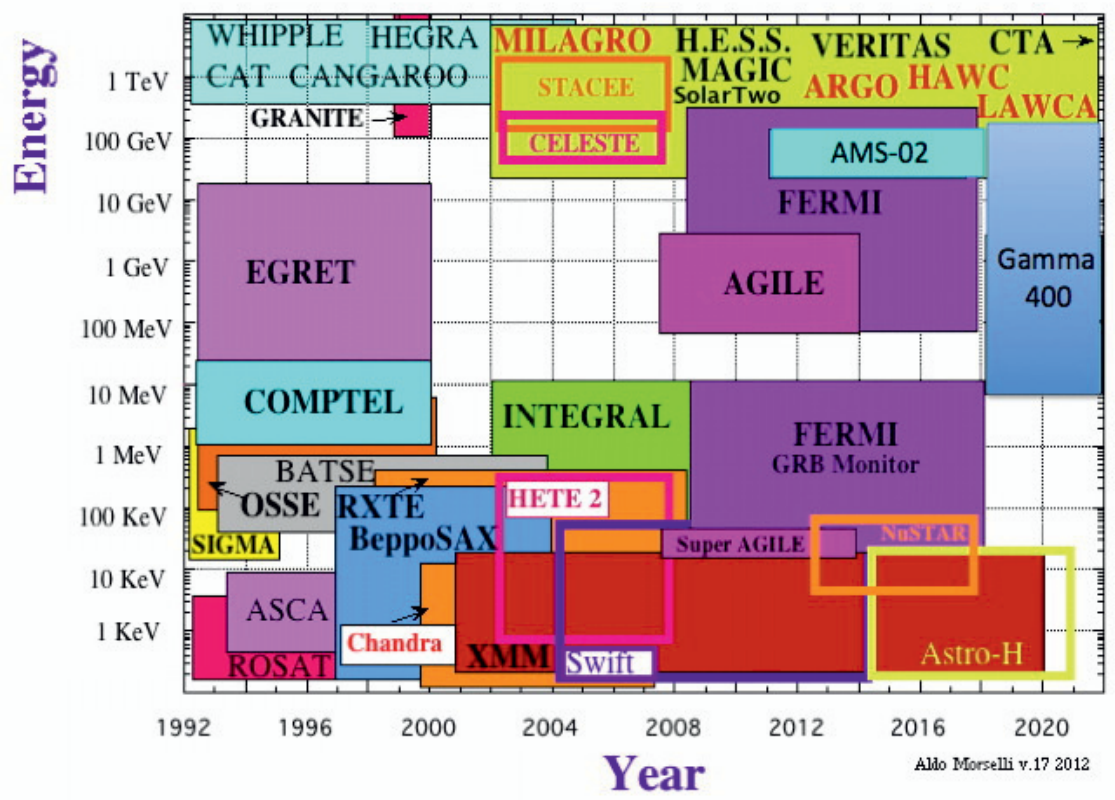

Figure 9. Timeline schedule versus the energy range covered by present and future detectors in $\mathrm{X}$ and gamma-ray astrophysics

\section{References}

[1] G.Basini, A.Morselli, M.Ricci, La Rivista del Nuovo Cimento, 12, n.4, pg.1-51 (1989)

[2] A.Morselli, The dark side of the Universe, pg.267-275, World Scientific, 1994

[3] W.B.Atwood et al. [Fermi Coll.], ApJ 697 (2009) 1071-1102 [arXiv:0902.1089]

[4] M.Ackermann et al. [Fermi Coll.], Astroparticle Physics 35 (2012) 346-353 [arXiv:1108.0201]

[5] M.Ackermann et al. [Fermi Coll.], ApJS 203 (2012) 4 [arXiv:1206.1896]

[6] A.A.Abdo et al. [Fermi Coll.], Physical Review Letters 102 (2009) 181101 [arXiv:0905.0025]

[7] M.Ackermann et al. [Fermi Coll.], Phys. Rev. D 82 (2010) 092004 [arXiv:1008.3999]

[8] M. Ackermann et al. [Fermi Coll.], Physical Review Letters 108, 011103 (2012) [arXiv:1109.0521 [astro-ph.HE]].

[9] O.Adriani. et al. [PAMELA Coll.], Physical Review Letters 106, p. 201101.

[10] O.Adriani. et al. [PAMELA Coll.], Physical Review Letters 111 (2013) 081102 [arXiv:1308.0133]

[11] D. Grasso, S. Profumo, A. W. Strong, L. Baldini, R. Bellazzini, E. D. Bloom, J. Bregeon, G. di Bernardo, D. Gaggero, N. Giglietto, T. Kamae, L. Latronico, F. Longo, M. N. Mazziotta, A. A. Moiseev, A. Morselli, J. F. Ormes, M. Pesce-Rollins, M. Pohl, M. Razzano, C. Sgro, G. Spandre and T. E. Stephens, Astroparticle Physics (2009) 32, 140.

[12] M.Ackermann et al. [Fermi Coll.], Physical Review D 82, 092003 (2010) [arXiv:1008.5119]

[13] M. Aguilar et al. [AMS-02 Coll.], Physical Review Letters 110, 141102 (2013)

[14] A. Morselli et al., Nucl.Phys. 113B (2002) 213 
[15] A.Cesarini, F.Fucito, A.Lionetto, A.Morselli, P. Ullio, Astropart. Phys. 21 (2004) 267 [astro$\mathrm{ph} / 0305075]$

[16] E. Baltz et al. , JCAP07 (2008) 013 [arXiv:0806.2911]

[17] V. Vitale and A. Morselli for the Fermi/LAT Collaboration, 2009 Fermi Symposium [arXiv:0912.3828]

[18] A. Morselli, B.Cañadas, V.Vitale, Il Nuovo Cimento 34 C, N. 3 (2011) [arXiv:1012.2292]

[19] G.Gomez Vargas et al., Journal of Cosmology and Astroparticle Physics JCAP 10 (2013) 029 [arXiv:1308.3515]

[20] A.Abdo et al. [Fermi Coll.] , ApJ 712 (2010) 147-158 [arXiv:1001.4531]

[21] M.Ackermann et al. [Fermi Coll.], Physical Review Letters 107 (2011) 241302 [arXiv:1108.3546]

[22] M.Ackermann et al. [Fermi Coll.], Physical Review D accepted [arXiv:1310.0828]

[23] Atlas Coll. JHEP 1304 (2013) 075 [arXiv:1210.4491]

[24] M.Ackermann et al. [Fermi Coll.], Physical Review D 86 (2012) 022002 [arXiv:1205.2739]

[25] A.Abdo et al. [Fermi Coll.], Phys. Rev. Lett. 104 (2010) 091302 [arXiv:1001.4836]

[26] C. Weniger, JCAP 1208 (2012) 007 [arXiv:1204.2797 [hep-ph]].

[27] M. Su and D. P. Finkbeiner, arXiv:1206.1616 [astro-ph.HE].

[28] T. Bringmann and C. Weniger, Dark Universe 1 (2012) 194-217 [arXiv:1208.5481]

[29] M.Ackermann et al. [Fermi Coll.], Physical Review D 88, 082002 (2013) [arXiv:1305.5597].

[30] see the slides presented at the "Science with Gamma-400 Workshop" http://www.fondazioneinternazionale.org/attiConvegni.php

[31] A.Morselli et al., Nuclear Physics B, Proceedings Supplements 2 39-240 (2013) 193-198 